\title{
Understanding the Li Production in AGB Stars: the J-type Stars
}

\author{
Carlos Abia \\ Dpt. Física Teórica y del Cosmos, Universidad de Granada, E-18071 \\ Granada, Spain \\ Jordi Isern \\ Institut d'Estudis Espacials de Catalunya-C.S.I.C., c/ Gran Capitá 2-4, \\ E-08034 Barcelona, Spain
}

\begin{abstract}
A full abundance analysis have been carried out in 12 galactic J-stars. The analysis shows: a) ${ }^{12} \mathrm{C} /{ }^{13} \mathrm{C}$ ratios are low, b) the abundance of s-process and rare-earth elements with respect to the stellar metallicity is nearly-solar, $[<$ heavy $>/ F e]=0.10 \pm 0.11 \mathrm{c}$ ) Tc is not present in most of the stars although upper limits are placed in WZ Cas and WX Cyg, probably two SC-type stars, and d) all the stars are Lirich, $\log \epsilon(\mathrm{Li})>1$. The location of J-stars in an empirical H-R diagram when compared with that of the normal N-type carbon stars, suggests that J-stars may be in the early-AGB phase. The different mechanism proposed to be responsible of this Li production are briefly discussed.
\end{abstract}

\section{Introduction}

J-stars were defined by Gordon (1971) as carbon stars showing very strong isotopic $\mathrm{CN} \& \mathrm{C}_{2}$ molecular bands implying usually very low ${ }^{12} \mathrm{C} /{ }^{13} \mathrm{C}$ ratios. There is observational evidence (e.g. Claussen et al. 1987) suggesting that most of galactic J-stars are low-mass objects, $\mathrm{M} \leq 2 \mathrm{M}_{\odot}$. Among the observed galactic Li-rich AGB stars more than 60\% are classified J-type (Boffin et al. 1993). However, current stellar models fail to produce low-mass, Li-rich AGB stars (see Sackmann, this volume). Indeed, the study of correlations between different chemical species might cast light on the Li production mechanism in these stars. Thus, we have performed an abundance analysis in 12 galactic J-type stars with known Hipparcos parallaxes, using high resolution and high $\mathrm{S} / \mathrm{N}$ spectra. Details about the observations and analysis can be found in Abia \& Isern (2000).

Figure 1 shows the location in the H-R diagram of the J-stars compared with normal $(\mathrm{N})$ carbon stars and R-type stars. From this figure one can conclude that $\mathrm{J}$-stars are in a transition phase between $\mathrm{R}$ - and N-type carbon stars. On the other hand, Figure 2 shows the $\mathrm{Li}$ vs. ${ }^{12} \mathrm{C} /{ }^{13} \mathrm{C}$ correlation found in our abundance analysis. Assuming that most of the J-stars studied here have $\mathrm{M} \leq 2$ $\mathrm{M}_{\odot}$, is it possible to explain all these chemical properties within the standard models of stellar evolution? 


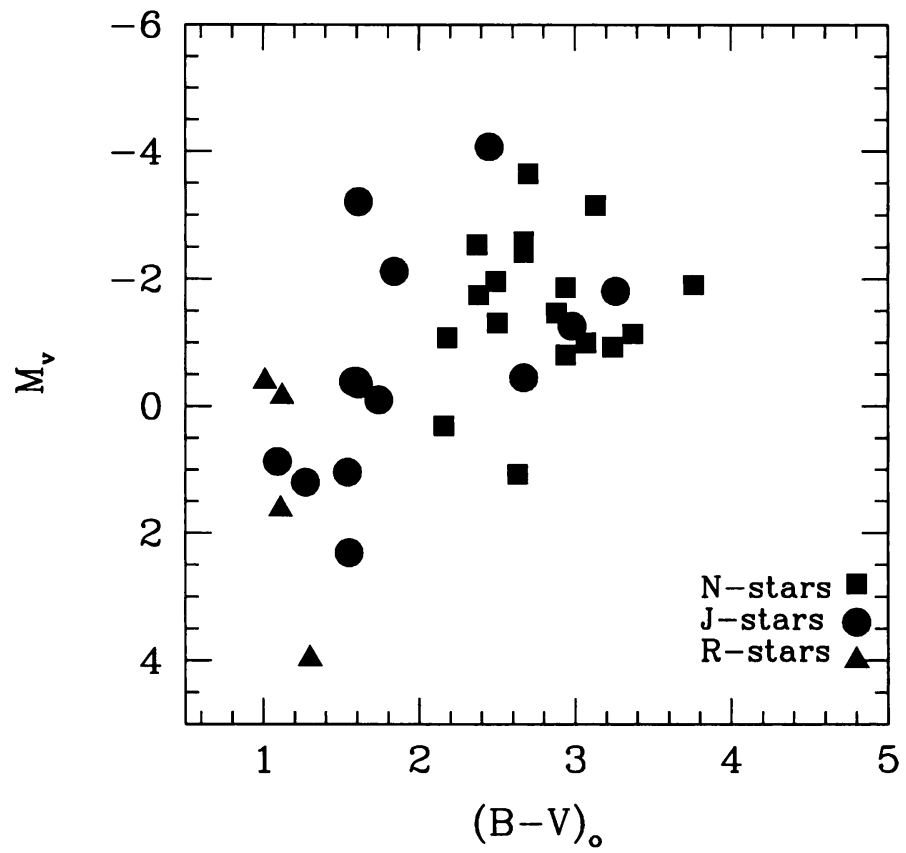

Figure 1. Observational H-R diagram for carbon stars. Data for Nand R-stars are taken from Alksnis et al. (1998).

\subsection{AGB stars?}

Current AGB models obtain C-rich $(\mathrm{C} / \mathrm{O}>1)$ and ${ }^{13} \mathrm{C}$-rich envelopes in stars with $\mathrm{M} \geq 4 \mathrm{M}_{\odot}$ through the operation of the successive He-shell flashes, $3^{\text {th }}$ dredge-up episodes and hot bottom burning. These stars can also be Li-rich stars for a long period of time. However, they should be fairly luminous, $\mathrm{M}_{\mathrm{bol}}<-6$ and present some s-nuclei enhancement. None of this is observed in the J-stars studied here. Furthermore, our objects are low-mass stars. Is there a nonstandard mixing mechanism and cool-bottom processing during the early AGB phase in low-mass stars? (see Sackmann's paper in this volume).

\subsection{Post He-core flash stars?}

Simulations of the He-core flash in giants by Deupree \& Wallace (1996) show that it is possible to mix enough core C-rich material into the envelope and transform the star into a carbon star. These authors claim that there is not s-nuclei production during the He-flash. However, no predictions are given concerning $\mathrm{Li}$ and ${ }^{13} \mathrm{C}$ production.

\subsection{Binary system?}

About $5-10 \%$ of J-star show silicate emission at $9.85 \mu \mathrm{m}$ which can be explained through the existence of an O-rich circumstellar envelope accumulated in a disc around an unseen hypothetical companion (Lloyd-Evans 1991). However, how could Li survive during the mass-transfer and posterior mixing? 


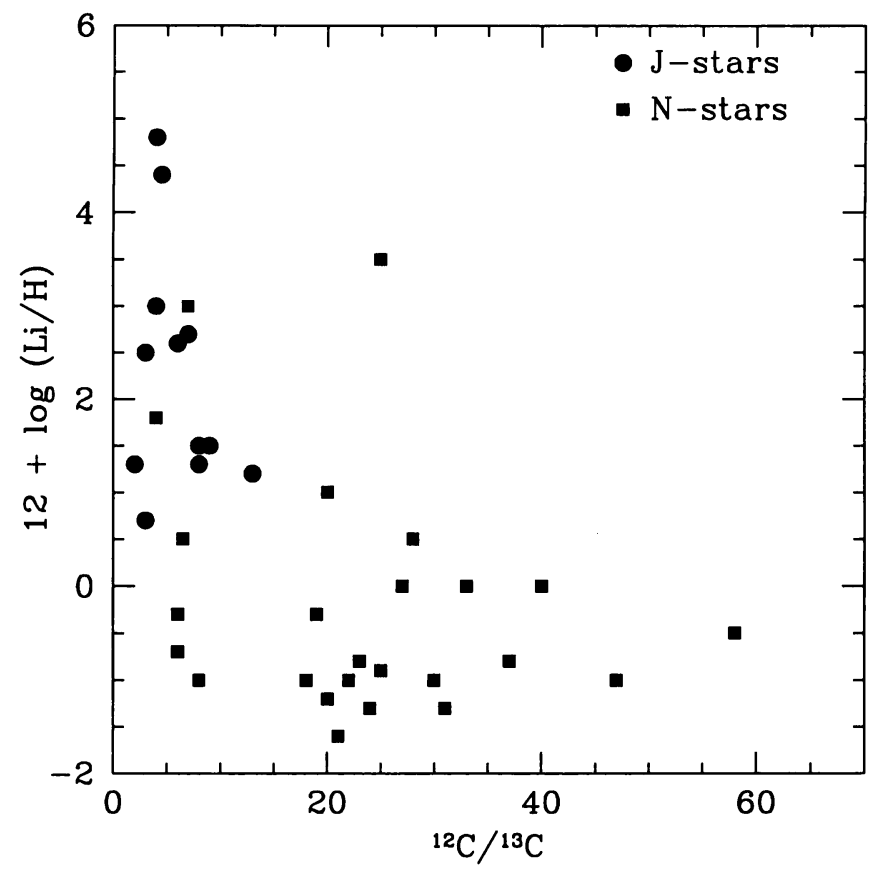

Figure 2. $\quad \mathrm{Li}$ abundances vs. ${ }^{12} \mathrm{C} /{ }^{13} \mathrm{C}$ in the carbon stars sample.

\section{Conclusions}

$\mathrm{J}$-stars are challenging objects for the theory of stellar evolution and in particular for the Li production in stars. No evolutionary scenario is able to explain all the observed chemical properties. Despite they are very frequently Li-rich objects, their contribution to the galactic $\mathrm{Li}$ is probably minimal due to their relatively low Li enhancements (see Abia \& Isern 2000) and mass-loss rates observed, $\sim 10^{-7} \mathrm{M}_{\odot} / \mathrm{yr}$. However, given the important uncertainties still existing in the derivation of the lithium abundance in carbon stars (Abia, Pavlenko \& de Laverny 1999), this conclusion should be considered with caution.

\section{References}

Abia, C., Pavlenko, Ya., \& de Laverny, P. 1999, A\&A, 351, 273

Abia, C., \& Isern, J. 2000, ApJ, (in press)

Alksnis, A., Balnlaus, A., Dzervitis, V. \& Eglitis, I. 1998, A\&A, 338, 273

Boffin, H.M.J., Abia, C., Isern, R., \& Rebolo, R. 1993, A\&AS, 102, 361

Claussen, M.J., Kleinmann, S.G., Joyce, R.R., \& Jura, M. 1987, ApJS, 65, 385

Deupree, R.G., \& Wallace, R.K. 1996, ApJ, 317, 214

Gordon, C.P. 1971, PASP, 83, 667

Lloyd-Evans, T. 1991, MNRAS, 249, 409 